\title{
Evidence of the persistence and consistency of social signatures
}

\author{
Yue Li and Robert M. Bond ${ }^{*}$ (D
}

\section{*Correspondence:}

bond.136@osu.edu

School of Communication,

The Ohio State University,

3072 Derby Hall, 154 North

Oval Mall, Columbus, $\mathrm{OH}$

43210, USA

\begin{abstract}
Human social networks are composed of multiple dynamic and overlapping communication networks, in which membership changes over time. However, less well understood are whether and how our communication patterns are similar or different over time and across various modes of communication. Here, we use data on the frequency of phone calls, text messages, and in-person interactions to examine the social signatures of more than 700 students in a university setting. Our analysis shows that although there is substantial turnover in participants' networks, participants' social signatures are persistent across time and consistent across communication modes. Further, we find that communication networks that are mediated via phone calls or text messages are more stable than are in-person networks. Our results show that, likely due to limitations in cognitive and emotional resources, people maintain networks of relatively stable size and structure their communication within those networks in predictable patterns. Our findings may help with formalizing social network theories, explaining individual-level attitudes and behaviors and aggregate-level social phenomena, and making predictions and detecting abnormalities in applied fields.
\end{abstract}

Keywords: Social signatures, Multi-modal network, Dynamic social network

\section{Introduction}

Social interactions play an important role in many aspects of human societies at both the individual level, such as mental health and individual well-being (House et al. 1988; Holt-Lunstad et al. 2010; Manninen et al. 2017), as well as the societal level, such as infectious disease control (Kafsi et al. 2013; Zhang et al. 2020) and viral marketing (Szabó and Barabasi 2003). Understanding how social interactions evolve over time and how they vary across different communication modes are essential. Without a deep understanding of temporal evolution of social interactions across communication channels, our understanding is limited in that we apply methods and models from studies of static networks to dynamic social processes, and from single modes of communication when social networks are frequently multi-layered. One approach to studying dynamic and multi-layered social interactions is from social network structures and their relationship to ego-centric tie strength. The distribution of tie strengths from an ego-centric perspective, as discussed in further detail below, has been dubbed an individual's "social signature" (Saramäki et al. 2014). That is, members of an inner layer (i.e.,

(c) The Author(s) 2022. Open Access This article is licensed under a Creative Commons Attribution 4.0 International License, which permits use, sharing, adaptation, distribution and reproduction in any medium or format, as long as you give appropriate credit to the original author(s) and the source, provide a link to the Creative Commons licence, and indicate if changes were made. The images or other third party material in this article are included in the article's Creative Commons licence, unless indicated otherwise in a credit line to the material. If material is not included in the article's Creative Commons licence and your intended use is not permitted by statutory regulation or exceeds the permitted use, you will need to obtain permission directly from the copyright holder. To view a copy of this licence, visit http:// creativecommons.org/licenses/by/4.0/. 
stronger tie strengths) of an individual's social contacts receive more communicative interactions and the outer layers (i.e., weaker tie strengths) receive less communicative interactions (Saramäki et al. 2014). These social structures, particularly when they are invariant across time and mode of network interaction within the same population, provide opportunities to examine fundamental network properties that enable the extension of theory. Social signatures may make individuals who are at the center of the social network receive information sooner than others (Garcia-Herranz et al. 2014), make it easier for individuals to live in "echo chambers" within which they mostly interact with others who hold similar beliefs, attitudes, and behaviors (Lewis et al. 2012), and accelerate the reinforcement of individuals' existing beliefs, attitudes, and behaviors through social influence (McPherson et al. 2001). These individual-level effects may evolve into an aggregate-level knowledge gap, attitudinal extremity and polarization, and health disparities. Thus, understanding whether and how social signatures change across communication channels, across individuals, and across time is essential to study both individual-level and aggregate-level social issues.

One gap in the literature about social network structures is that researchers have paid substantially more scholarly attention to varying structural properties than to invariant properties. Social signatures are the allocation of social resources across members of a focal individual's social network, which may vary over communication modes and over time. Researchers have tended to assume that different social networks have varying network structural properties (Borgatti et al. 2009). These variations in social network structures across people, channels, and time could then explain differences in outcomes, such as imbalanced information flow and polarized attitudes and behaviors. However, we argue that exploring invariant social network structures may be similarly important for both formalizing social network theories and applying social network theories to explain applied social network-related phenomena. First, exploring invariant social network structures can help with formalizing social network theories. One criticism of social network research is that the field tends to be "merely descriptive" or "just methodology" (Borgatti et al. 2009). Examining invariant underlying structural components of social networks across people, channels, and time may help with formalizing social network theories and increasing their explanatory power. Second, invariant structures of social networks can help explain the variance in outcomes across social networks. Individual differences indeed explain a certain amount of variance in outcomes. Yet, structurally equivalent roles (i.e., people in similar positions and that have similar ties in a network) rather than individuals may help explain more variance in the outcomes (Borgatti et al. 2009) and thus increase the generalizability of findings. Structurally equivalent individuals may face similar environments in which they may gradually develop similar responses, such as similar attitudes and behaviors.

Understanding the fundamental invariant structures of social interactions has been a central challenge for scholars (Sekara et al. 2016), which has highlighted two major opportunities for advancing our understanding in this area. A fundamental question in this area is: invariant structures relative to what? On the one hand, it may be invariant structures of social interactions across differing channels of interaction. An increasing number of information and communication technologies make it possible for people to interact with each other through multiple channels (Mones et al. 2017), 
from person-to-person interactions (Cattuto et al. 2010), via phone calls (Onnela et al. 2007), and through social media (Grabowicz et al. 2012), among other modes. Social interactions across channels are inherently overlapping (Palla et al. 2005; Ahn et al. 2010; Rosvall et al. 2014). A frontier in this area is multi-layer communication networks, and particularly the temporal stability of interaction patterns across modes of communication. Studies in this area are challenging because of the intrinsic differences between different communication channels (Heydari et al. 2018), including measurement challenges surrounding whether and how to compare or combine weighted and unweighted communication network measures (Saramäki and Moro 2015). On the other hand, it may be that patterns of social interactions are invariant across time, but may or may not vary over communication mode. Understanding meso-level social group behaviors (Palla et al. 2007; Lin et al. 2009) and their temporal evolution or lack thereof across time is essential in understanding the time-invariant fundamental structures and regularities governing social groups (Holme and Saramäki 2012; Sekara et al. 2016).

To enable an in-depth understanding of the channel- and time-invariant characteristics of social network structures, we focus on dynamic multi-layer social networks across communication modes (i.e., person-to-person, phone calls, and text messages) and across time. We note that the communication modes vary in both physical proximity, with in-person interactions likely being relatively proximate compared to phone calls and text messages, and synchronicity, with in-person interactions being synchronous, phone calls being sometimes synchronous and sometimes not (e.g., because of a voicemail message), and text messages being asynchronous (although sometimes alternating in a short period). Therefore, we categorize social networks into person-to-person networks and mediated networks (i.e., phone call and text message). Our study aims to examine the persistence of social signatures across time in both person-to-person networks and mediated networks. We also aim to compare the consistency of social signatures across different communication modes, including both mediated channels and in-person channels. Specifically, we analyze the complete dynamic multi-layer social networks collected by the Copenhagen Networks Study (Sapiezynski et al. 2019) (CNS) to explore: (1) how individuals allocate their communication resources across people (i.e., individuals' social signatures) in the person-to-person (in-person interactions) and mediated networks (i.e., phone call and text message), (2) whether a given individual's social signature is persistent over time, but different from other individuals' social signatures, and (3) whether a given individual's social signatures in different communication modes are similar to each other.

Our work advances the literature in at least three ways. Theoretically, we provide a broader understanding of social signatures by comparing and incorporating multimodal social networks. Previous research on social signatures primarily has investigated mediated networks (Saramäki et al. 2014; Heydari et al. 2018). We analyze person-toperson networks in addition to mediated networks and show that social signatures are consistent across different communication modes, from person-to-person to mediated networks. Additionally, we show the existence of persistence of social signatures in the so far largest multi-layer social network used to examine social signatures with more than 780 participants. By doing so, we substantially improve the generalizability of previous research on the persistence of social signatures. We also show that the time period 
to observe persistence of social signatures could be shortened to within one month whereas prior studies have examined longer time periods. Finally, our study has implications for empirical studies and may help with formalizing social network theories.

To study the consistency and persistence of social signatures across communication modes, we analyze a previously published longitudinal data set that includes social network interactions across phone calls, text messages, and in-person interactions detected by Bluetooth (Sapiezynski et al. 2019). These data include participants' interactions across communication modes and across time such that we are able to construct social signatures for participants both within a given mode of communication and by combining the modes into a single multi-channel network, as described below. Using these data, we compare the social signatures of individuals across communication modes to reference distributions to examine whether the social signatures of individuals are consistent across communication modes. We also compare the distance between two social signatures of each focal ego at two different time periods to the distance between the social signatures of the focal ego and a randomly selected other participant. This enables us to examine whether the social signatures of individuals are persistent across time. This work takes inspiration from prior work on social signatures (Saramäki et al. 2014), including work that examines multiple modes of interaction (Heydari et al. 2018).

The structure of the paper is as follows. The next two sub-sections review the literature on static and dynamic social signatures across different communication modes. The subsequent section describes the data sets and summarizes our analytical methods. The three sub-sections under the results section summarize our results about social signatures from three aspects: turnover rate, persistence, and consistency. We then discuss the implications of our findings and the limitations of our study in the discussion section.

\section{Static and dynamic social signatures}

Existing research has examined patterns of both static and dynamic social interactions through single or multiple communication channels. One key finding in static social networks is that social interactions are constrained by cognitive limits. According to this theory, the maximum number of relationships an individual can actively maintain averages approximately 150 contacts (Hill and Dunbar 2003; Dunbar 2014). This hypothesis rests on the assumption that maintaining social relationships is costly in terms of time (Miritello et al. 2013; Milardo et al. 1983), cognitive resources (Stiller and Dunbar 2007; Powell et al. 2012), and emotional capability (Saramäki et al. 2014). Within the set of 150 people, the first and closest circle, usually called an individual's support clique, is limited to approximately five members (Dunbar and Spoors 1995). More distant circles include the sympathy group ( $\sim 15$ members), the affinity group ( $\sim 50$ members), and the active network ( $\sim 150$ members). Although the basis of this theory is rooted in offline social networks (Hill and Dunbar 2003), support for groups of approximately this size have been found in mobile phone networks (Mac Carron et al. 2016), social networking sites (Arnaboldi et al. 2013; Dunbar et al. 2015), and online computer games (Fuchs et al. 2014). Indeed, these findings are intriguing as they suggest that the number of friends at a given time for each individual in a single communication mode approximates the 150-person theoretical average. Yet, static social network analysis hinders detailed understanding of the mechanisms shaping the underlying social network structure by 
focusing on a single temporal slice (Laurent et al. 2015) and a single communication mode. Even within the relatively static groups that make up an individual's social network, there may be meaningful variation in how much attention is paid to a given social contact over time or across communication modes. Therefore, social networks need to be examined in a dynamic setting across different communication modes within an individual.

Irrespective of the different communication channels that individuals use to interact with each other, research must advance our understanding of whether the social interaction patterns observed in static social networks change or persist over time for each individual. Social networks may change over time depending on many factors, such as job transition, relocation, adoption of new information communication technologies, or simply age. Despite these changes, research has found that the way in which individuals allocate their communicative effort among members of their social networks is persistent over time (Saramäki et al. 2014). Within the general pattern, there are between-person variations so that each individual has a distinct social signature. The persistence of social signatures was first found in a phone call network (Saramäki et al. 2014) and similar social signatures have been found in text message networks (Heydari et al. 2018) and online social networks (Liu et al. 2018; Koltsova et al. 2021).

\section{Social signatures across different communication modes}

Though both static and dynamic patterns in a single channel uncover valuable characteristics of social interactions, comparing these patterns across different communication modes may provide more insight into the underlying structures and mechanisms of social interactions. More importantly, cross-channel comparisons make it possible to examine whether the persistence of social signatures is a genuine characteristic of egocentric social networks and can be generalized to other communication modes. Some work on social signatures has found that mediated networks (i.e., call networks and text messaging networks) are similar and display patterns that are distinct from the personto-person network (Stopczynski et al. 2014), but other work has found that for a given individual, the text message patterns and phone call patterns may differ significantly with respect to daily communication patterns, indicating that phone calls and text messages serve different roles in communication (Aledavood et al. 2016). Despite these differences among different communication modes, scholars have also found similarities. Individuals have similar egocentric network structures in both online and offline social networks (Arnaboldi et al. 2013). Social signatures in mediated communication networks (i.e., phone calls, and text messages) show persistent individual differences over time, despite the turnover in each individual's networks (Heydari et al. 2018). A more integrated approach to comparing and integrating in-person and mediated social networks in an individual's life would greatly advance our knowledge of human interactions.

Integrating a given individual's social networks into one network may help correct the biases introduced by observing single channels. There are two main concerns related to the single-channel approach (Stopczynski et al. 2014). First, the single-channel approach only captures an incomplete picture of the entire life of an individual. That is, the observations based on one communication mode will provide incomplete samples of a complete network (Nanavati et al. 2008; Wang et al. 2013). Human social interactions 
between the same individuals take place across many different communication channels, such as face-to-face communication, phone calls, text messages, emails, social networking sites, and many other platforms. In some instances, the networks composed by these various modes of communication will overlap and in others they will not. We must study the communication channels that are typically examined separately together to fully understand human patterns of interaction (Stopczynski et al. 2014). Second, the single-channel approach may limit interdisciplinary cooperation (Stopczynski et al. 2014), which may inhibit our ability to have a broad theoretical understanding of which relationships are specific to a given communication mode and which are more universal. Scholars using single-channel approaches may find it difficult to validate their findings in different channels that could be useful to scholars in other disciplines. The limitations of the single-channel approach can be reduced by using a multi-channel approach. To our knowledge, scholars who have integrated different social networks to study social signatures have mainly examined mediated networks, such as phone call networks and text message networks (Heydari et al. 2018), perhaps due to the relative convenience of collecting such data. We use the Copenhagen Network Study that enables the integration of in-person networks and mediated networks (Stopczynski et al. 2014; Mones et al. 2017; Sekara et al. 2016; Alessandretti et al. 2018; Sapiezynski et al. 2018). Scholars using this data have found that relationships in mediated settings, such as Facebook interactions, phone calls, and text messages, are frequently reflected in offline behaviors (Sapiezynski et al. 2018) and that central members in the offline network interact with the environment regularly, whereas core members in the online networks show irregular behaviors and are more active in irregular social activities (Mones et al. 2017). These data have the substantial potential to be used to examine a key component of multi-modal communication networks, namely whether social signatures persist both in time and across communication modes. We note that another researcher independently and contemporaneously used the same data set to study a related set of questions (Schulman 2021).

\section{Materials and methods}

The data examined in this study come from the CNS. The CNS has the largest and the most dense population to date among studies analyzing multi-layer high-resolution social networks. The original researchers collected temporally dynamic, multi-layer network data from a densely interconnected cohort of 787 newly-admitted undergraduate freshmen students at the Technical University of Denmark. 78\% of the participants are male and 22\% are female. Each participant was equipped with an Android smartphone, through which Bluetooth data were collected every five minutes, and phone call and text message data were collected over the observation period regardless of the participants' activities. The total observation time used in this study was four weeks, starting from a Sunday during a school term. The time period observed is the same across all students. The Bluetooth data can be used as a proxy for physical distance, which is a reasonable proxy for person-to-person interactions (though see a discussion of the limitations of this approach below). The phone call and text message data represent mediated communication through cellphones, with which participants were equipped for the purposes of the study. 
To compare and integrate the networks, we then use the approach developed by Heydari et al. (2018). The challenge in comparing and integrating the three networks is defining the weights of ties in the networks because the frequencies of phone calls, text messages, and in-person interactions cannot be directly compared. We break the timeline of each egoalter interaction and divide it into time bins of $1 \mathrm{~h}$ across the observation period. We then count the number of bins that contain at least one social interaction between an ego and alter. These counts are used as the weights for ties between egos and alters. For example, a given individual (i.e., the ego in Fig. 1) has six contacts in total in a random day. For the specific node $\mathrm{E}$, the ego texted with $\mathrm{E}$ during $2 \mathrm{~h}$, called $\mathrm{E}$ during $3 \mathrm{~h}$, and was with $\mathrm{E}$ in person during $1 \mathrm{~h}$. Therefore, the total time of interactions will be five no matter how many times the ego texts, calls, and sees the node E. As illustrated by the example, if a given node had a text message conversation that went back and forth multiple times all during the same hour, that would be measured as a single hour of interaction. Using this approach, we integrate the phone call network, the text message network, and the person-to-person network. In doing so, we are able to investigate the three single-mode communication networks as well as an integrated multi-mode network.

To measure the consistency and persistence of social signatures, the first step is to examine whether the composition of the social networks changes across time, individuals, and channels. Examining changes in the composition of each ego's social network members helps to inform further examinations of social signatures. If ego networks have low overlap but still have high consistency in terms of social signatures that would have a different interpretation than if both the overlap of network members and social signature patterns remained relatively stable. Therefore, our first measure is Jaccard Index (JI), which measures the overlap of two sets of alters in a pair of egocentric networks (Heydari et al. 2018; Saramäki et al. 2014). The JI is defined as:

$$
J\left(\sigma_{1}, \sigma_{2}\right)=\frac{\left|\sigma_{1} \bigcap \sigma_{2}\right|}{\left|\sigma_{1} \bigcup \sigma_{2}\right|}
$$

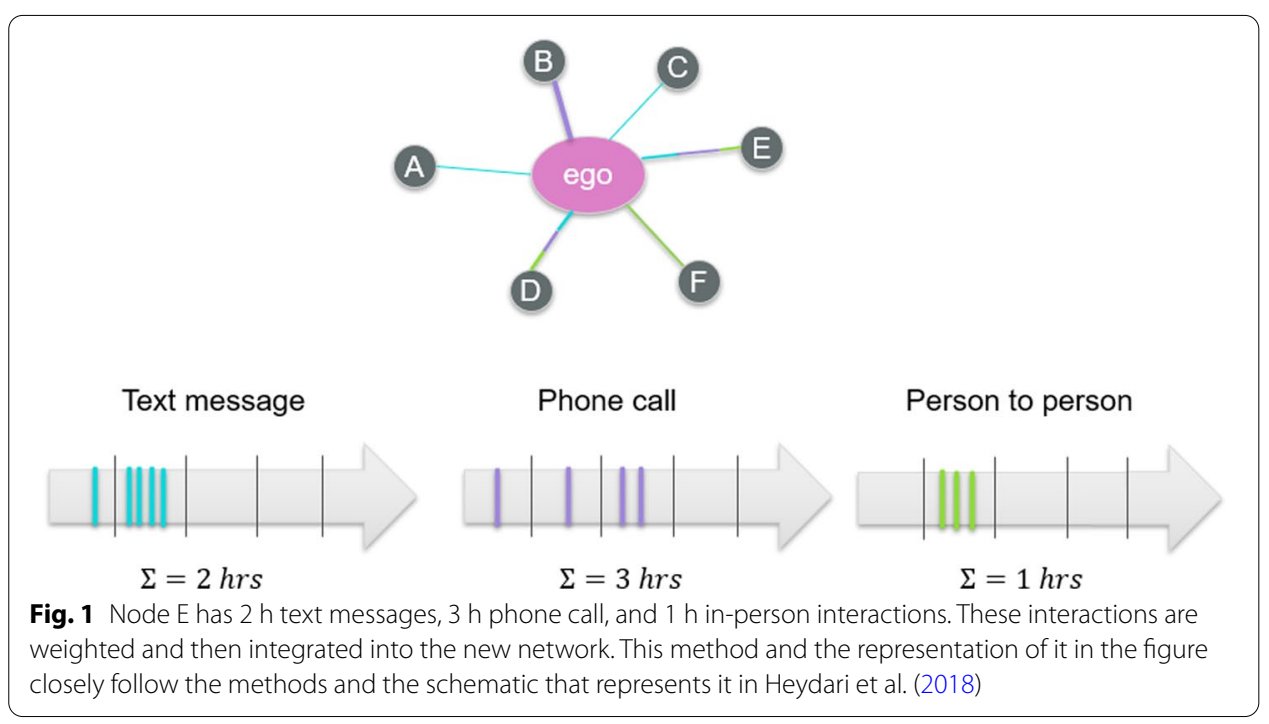


where $\sigma_{1}$ and $\sigma_{2}$ are two social signatures. If there is complete overlap between the two sets of alters, then $\mathrm{JI}=1$; if there is no overlap between the two sets of alters, then $\mathrm{JI}=0$.

The next step is to compare whether the underlying structures of the social networks change. To do that, our second measure is Jensen-Shannon distance (JSD), which measures how a pair of probability distributions diverge from each other. JSD has previously been used by network researchers to measure the similarity between two social signatures (Heydari et al. 2018; Saramäki et al. 2014). The JSD is defined as:

$$
J S D\left(\sigma_{1}, \sigma_{2}\right)=H\left(\frac{1}{2} \sigma_{1}+\frac{1}{2} \sigma_{2}\right)-\frac{1}{2}\left[H\left(\sigma_{1}\right)+H\left(\sigma_{2}\right)\right]
$$

where $\sigma_{1}$ and $\sigma_{2}$ are two social signatures, $H(\sigma)$ is the Shannon entropy of $\sigma$. Thus, JSD is minimized when the probability distributions are the same and becomes larger as they diverge.

We then combine these two measures to answer our research questions. First, to examine the persistence of social signatures (i.e., whether individuals' social signatures stay stable across time), we divide the 28-day observation into two intervals $I_{1}$ and $I_{2}$, each of which encompasses 14 days. We compare social signatures using a 14-day time interval rather than on a daily- or weekly- basis such that we are able to capture the underlying social signatures rather than random noise due to daily life. By splitting the observed period into the two largest, equally sized time periods possible, we limit the impact that any given day, day of week, and so on, would have on our measurements while still enabling a time-dynamic comparison of equally sized periods of time. Previous studies have analyzed the persistence of social signatures in a similar way (Saramäki et al. 2014; Heydari et al. 2018). Further, as discussed in detail below, understanding whether persistent social signatures may be observed in relatively short periods of time is important for future research in this area. We then calculate a given individual's JI between $I_{1}$ and $I_{2}$ to see whether a given individual's social networks members change over time. We then compare a given individual's JSD between $I_{1}$ and $I_{2}$ with the JSD of random participant in $I_{1}$ and the JSD of a random participant in $I_{2}$ to see whether individuals' social signatures stay stable over time. We repeat the same procedure for all four social networks (i.e., in-person, text message, phone call, and integrated network).

Second, to examine the consistency of social signatures (i.e., whether individuals' social signatures stay stable across communication modes), we compare a given individual's JI between in-person and text message networks, between in-person and phone call networks, and between text message and phone call networks, to examine whether the members of the same person's social networks stay the same. We then compare a given individual's JSD between his or her own social networks with the corresponding JSD between the individual and a random other. We do so to examine whether individuals' social signatures stay stable across different communication modes. We repeat the procedure for all paired combinations between in-person, text message, and phone call networks.

\section{Results}

\section{Turnover in individual social signatures}

The distribution of weights in human social signatures in the person-to-person, phone call, text message, and integrated social network appear to be relatively broad, 
as seen in Fig. 2. A small number of contacts of an individual participate in the majority of interactions, whereas most of the contacts only participate in a small amount of social interactions of that individual. This holds true over time and across all of the four social networks. To quantify the level of turnover in the networks, we compared the composition of alters in $I_{1}$ and $I_{2}$ using the Jaccard Index. A high level of turnover within each individual's network would be indicated by low Jaccard Index score; a low level of turnover within each individual's network would be indicated by high Jaccard Index score, as illustrated in Fig. 3. For the person-to-person network of participants, $J\left(I_{1}, I_{2}\right)=0.33 \pm 0.01$; for the phone call network, $J\left(I_{1}, I_{2}\right)=0.44 \pm 0.02$; for the text message network, $J\left(I_{1}, I_{2}\right)=0.51 \pm 0.02$. For the integrated network that combined the three networks, $J\left(I_{1}, I_{2}\right)=0.34 \pm 0.01$. The results indicate that the turnover rate is the largest for the person-to-person network, followed by the phone call network, and the turnover rate is the lowest for the text message network. For the combined network, the turnover rate is most similar to that of the person-to-person network. The differences between the distributions shown in Fig. 3 are consistent with expectations given the nature of the data. Given that the person-to-person interactions observed are due to proximity measured by Bluetooth, we expect that turnover may be relatively high as these are a mixture of purposeful interactions, structured interactions (such as being in

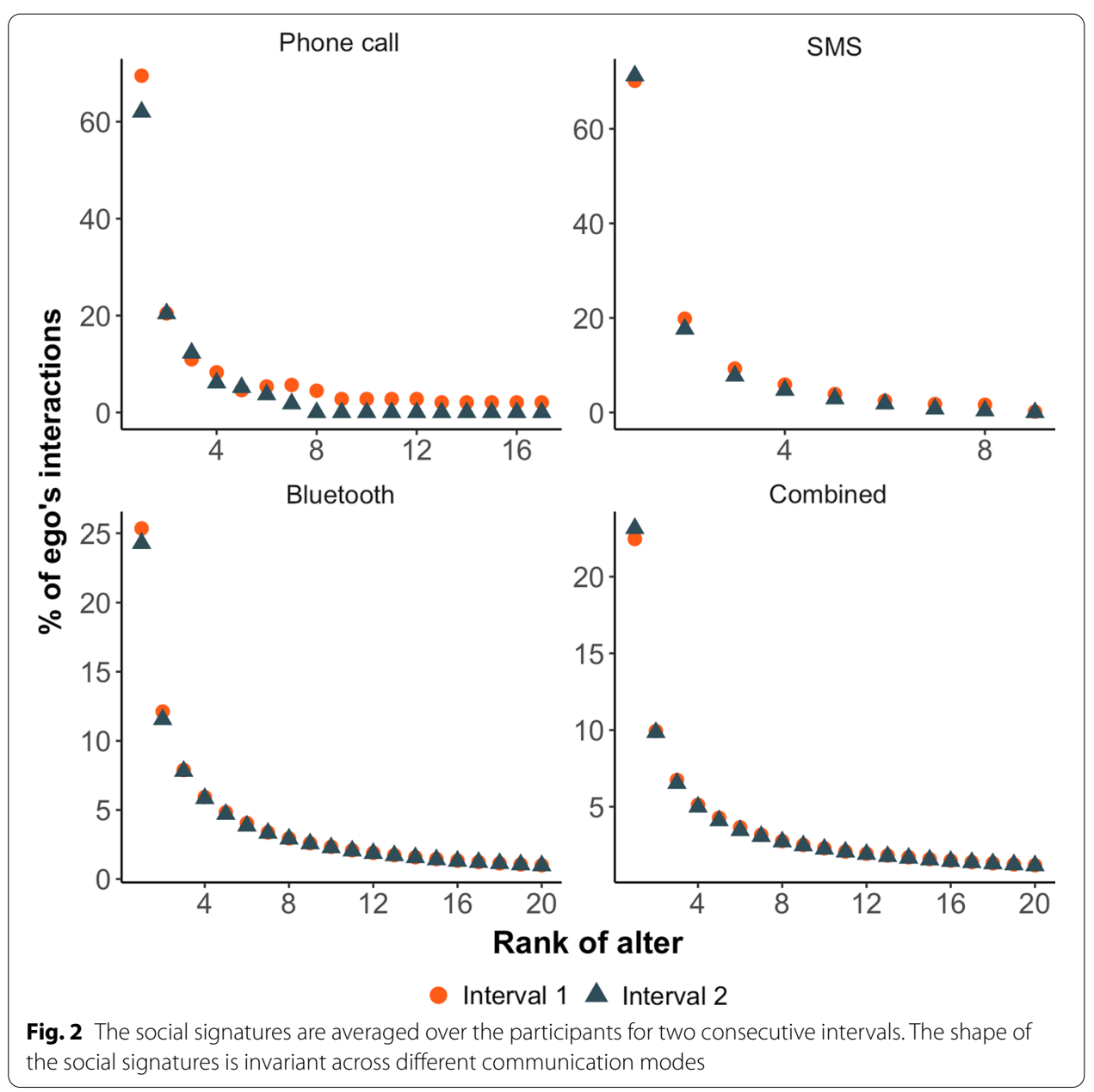




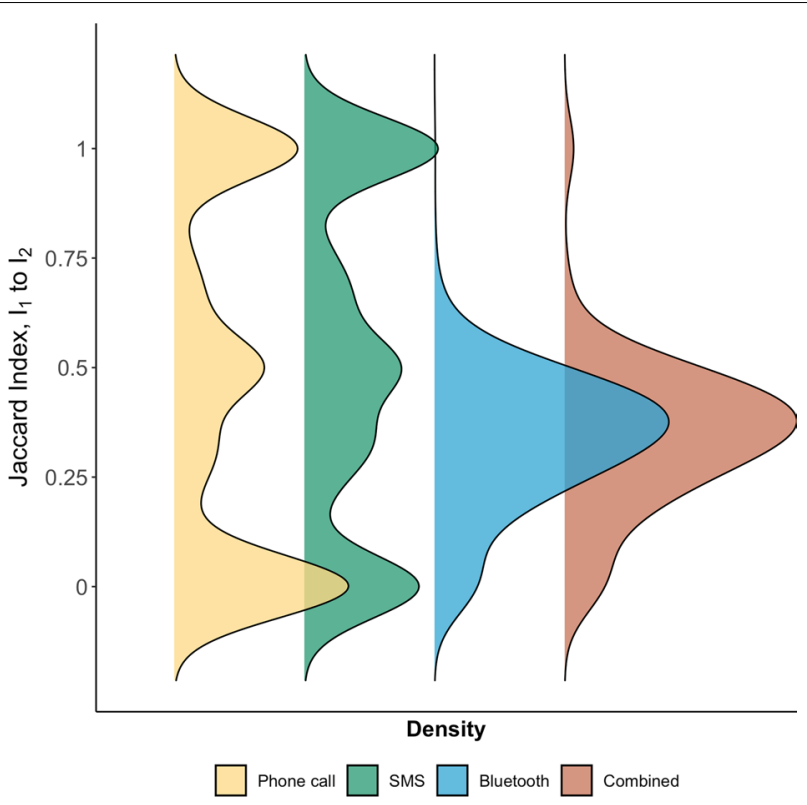

Fig. 3 The Jaccard Indexes are averaged over the participants for two consecutive intervals. The high turnover rates in the person-to-person network and the combined network are reflected as the larger Jaccard Indexes between the two intervals in the two networks

a shared classroom) and chance encounters. The phone call and SMS networks are much more likely to be purposeful interactions among relatively strong ties. In all cases, the results suggest that there is substantial turnover in the set of people participants interacted with between $I_{1}$ and $I_{2}$.

\section{Persistence of individual social signatures}

To examine the persistence of individual social signatures, we compared the JensenShannon Divergence (JSD) between $I_{1}$ and $I_{2}$ for each network. We calculated ego $i$ 's self-distances between $I_{1}$ and $I_{2}$, denoted as $d_{\text {self }}$. A low self-distance score indicates high similarity between the ego's social signatures in the two intervals. We then calculated reference distances using two approaches: first, we randomly selected another participant in $I_{1}$ and calculated the distance between the signatures of the ego $i$ and the signatures of the randomly selected other participant in $I_{1}$, denoted as $d_{\text {ref } 1}$; second, we randomly selected another participant in $I_{2}$ and calculated the distance between the signatures of the ego $i$ and the signatures of the randomly selected other participant in $I_{2}$, denoted as $d_{r e f 2}$. In doing so, we are able to compare the distributions of $d_{\text {self }}$ to both $d_{r e f 1}$ and $d_{r e f 2}$. If $d_{\text {self }}$ is significantly smaller than the reference distributions this would suggest that an individual's social signature is more stable over time than we would expect from chance.

The results show that on average, the shapes of the social signatures are persistent over time, as seen in Fig. 4. Specifically, we found that on average, the distance between an individual's social signatures in two consecutive intervals is significantly smaller than the distance between the individual's social signature and the social signatures of another random individual either in $I_{1}$ or $I_{2}$. The finding holds true across all three networks. Specifically, for the person-to-person network, the average self-distance is $d_{\text {self }}=0.075 \pm 0.005$ whereas the average distance to a random individual in 


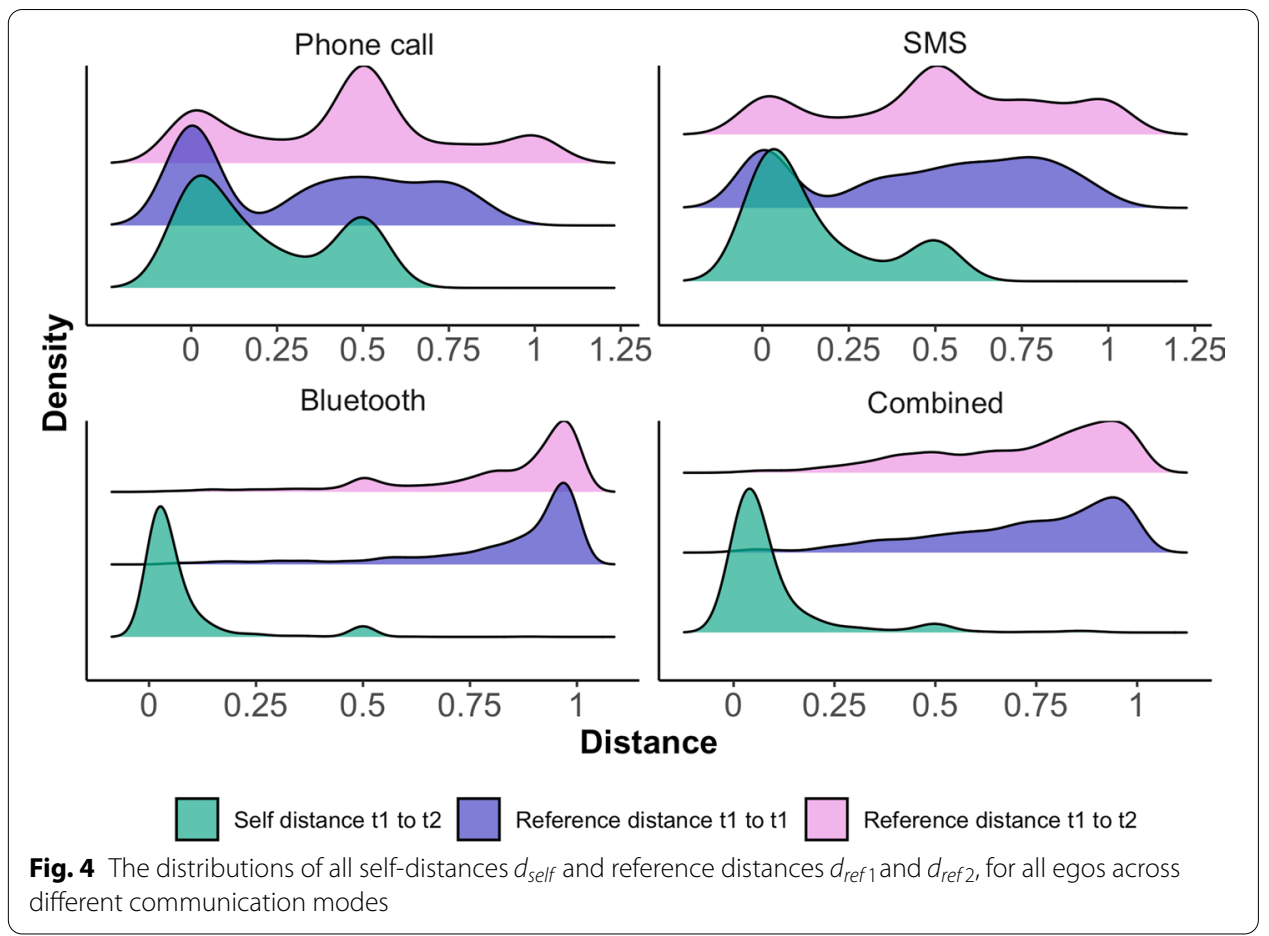

$I_{1}$ is $d_{r e f 1}=0.828 \pm 0.008$ and the average distance to another random individual in $I_{2}$ is $d_{r e f 2}=0.810 \pm 0.008\left(t_{I 1}(1019)=-77.00, p<.001 ; t_{I 2}(1004)=-73.78, p<.001\right)$. Similarly, for the phone call network, the average self-distance is $d_{\text {self }}=0.207 \pm 0.012$ whereas the average distance to a random individual in $I_{1}$ is $d_{r e f 1}=0.309 \pm 0.017$ and the average distance to another random individual in $I_{2}$ is $d_{r e f 2}=0.437 \pm 0.016\left(t_{I 1}\right.$ $\left.(510)=-4.75, p<.001 ; t_{I 2}(519)=-10.89, p<.001\right)$. Next, for the text messages network, the average self-distance is $d_{\text {self }}=0.151 \pm 0.009$ whereas the average distance to a random individual in $I_{1}$ is $d_{r e f 1}=0.467 \pm 0.017$ and the average distance to another random individual in $I_{2}$ is $d_{r e f 2}=0.519 \pm 0.016\left(t_{I 1}(605)=-16.286, p<.001\right.$; $\left.t_{I 2}(615)=-19.317, p<.001\right)$. Finally, for the combined network, the average of self-distance is $d_{\text {self }}=0.10 \pm 0.005$ whereas the average distance to a random ego in $I_{1}$ is $d_{r e f 1}=0.71 \pm 0.009$ and the average distance to another random ego in $I_{2}$ is $d_{\text {ref } 2}=0.71 \pm 0.009\left(t_{I 1}(1112)=-58.689, p<.001 ; t_{I 2}(1123)=-58.806, p<.001\right)$.

\section{Consistency of social signatures across communication modes}

We next compared the alter composition of the three networks (i.e., person-to-person network, phone call network, and text message network). We found that with regard to the composition of alters, the phone call network and the text message network are more similar to each other, as seen in Fig. 5 compared with the person-to-person network $(J(s m s, b t)=0.002 \pm 0.002 ; \quad J($ call,$b t)=0.007 \pm 0.001 ; \quad J(s m s$, call $)=0.455 \pm 0.017 ;$ $t_{\text {sms.callvs.sms.bt }}(533)=-27.185, p<.001 ; \quad t_{\text {sms.call vs. call.bt }}(528)=-26.966, p<.001$; $\left.t_{\text {sms.bt vs. call.bt }}(1134)=-2.244, p<.05\right)$. In other words, individuals interact with primarily the same group of people through phone calls or text messages. When they switch from mediated communication to person-to-person communication, individuals usually interact with a different group of people. As discussed in greater detail below, 


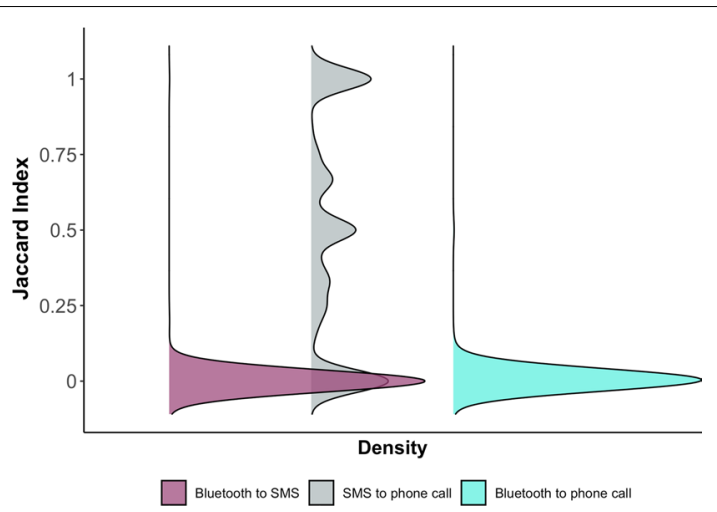

Fig. 5 The Jaccard Indexes are averaged over the participants for each pair of comparison among the three networks. The similarity of alter composition between the text message network and the phone call network is indicated by the higher Jaccard Index between SMS and phone call

this is again consistent with the likelihood that for many participants mediated communication is more purposeful and likely to be directed primarily at strong ties while in-person interactions (as detected by Bluetooth) are likely to be a mixture of purposeful interactions and circumstantial, non-social interactions. We then compared the distances between each pair of the three networks within a given individual with the distances between the individual and another randomly selected individual within the same network. We found that the social signatures are significantly consistent within people across different communication channels, as illustrated in Fig. 6. Specifically, the average distance between a given individual's social signature in the person-to-person network and the social signature in the text message network is significantly smaller than both the distance between the same individual's social signature and a random other's social signature in the person-to-person network $\left(d_{b t s m s / \text { self }}=0.509 \pm 0.007\right.$, $\left.d_{\text {btsms/other.bt }}=0.842 \pm 0.008, t(1222)=-30.92, p<.001\right)$ and the distance between the same individual's social signature and a random other's social signature in the text message network ( $\left.d_{b t s m s / o t h e r . s m s}=0.747 \pm 0.012, t(1013)=-16.86, p<.001\right)$. For the comparison between the text message network and the phone call network, the average distance between a given individual's social signature in the text message network and the social signature in the phone call network is significantly smaller than both the distance between the same individual and a random other in the text message network $\left(d_{\text {smscall/self }}=0.191 \pm 0.009, \quad d_{\text {smscall/other.sms }}=0.524 \pm 0.015\right.$, $t(830)=-19.12, p<.001)$ and the distance between the same ego and a random other in the phone call network $\left(d_{\text {smscall/other.call }}=0.543 \pm 0.014, t(886)=-21.89, p<.001\right)$. For the comparison between the person-to-person network and the phone call network, the average distance between a given individual's social signature in the person-to-person network and the social signature in the phone call network is significantly smaller than both the distance between the same individual's social signature and a random other's social signature in the person-to-person network $\left(d_{\text {btcall } / \text { self }}=0.512 \pm 0.007, d_{\text {btcall } / \text { other.bt }}=0.858 \pm 0.007, t(1220)=-35.10, p<.001\right)$ and the distance between the same individual's social signature in the phone call network $\left(d_{\text {btcall } / \text { other.call }}=0.633 \pm 0.014, t(888)=-8.01, p<.001\right)$. 


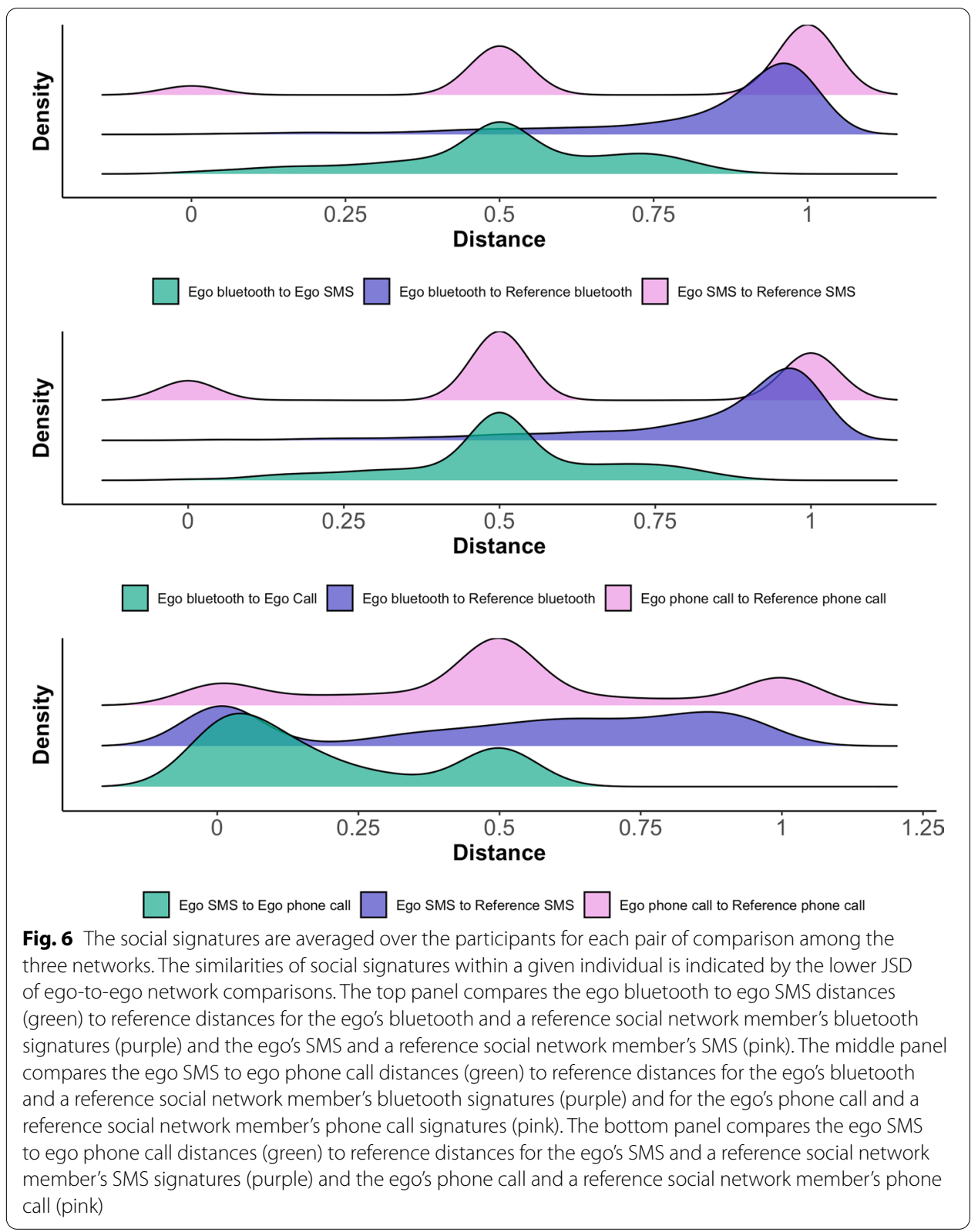

\section{Discussion}

This study provides a broader and deeper understanding of the characteristics of human social signatures in several ways: (1) individuals have consistent and unique ways of allocating communication resources (i.e., social signatures) across the members of different social networks; (2) the mediated communication networks (i.e., the phone call network and text message network) display substantially distinct characteristics from the person-to-person network. First, the mediated communication networks have a relatively low turnover of their members compared to the person-toperson network. Second, with respect to the composition of alters, the two mediated social networks are relatively similar, whereas the person-to-person network has less 
overlap with the mediated communication networks; (3) most importantly, the social signatures of all three social networks remain persistent over time, even with high turnover. Each individual's social signatures across different communication modes are similar to each other. In sum, individuals' social signatures are persistent across time and consistent across communication modes.

The patterns of social interaction demonstrate that human communities are inherently overlapping with each individual participating in different social networks contemporaneously (Saramäki et al. 2014; Sekara et al. 2016; Sapiezynski et al. 2018). The social interaction patterns across various channels differ to an extent that individual social ties cannot be reduced to a single layer. Recent scholarship has focused on the differences of social interaction patterns across different channels (Stopczynski et al. 2014; Aledavood et al. 2016; Mones et al. 2017; Heydari et al. 2018; Sapiezynski et al. 2018). Most studies have compared multi-channel high-resolution data without considering the nature of different communication modes and the synchronicity of human interactions. Media richness theory suggests that individuals choose different communication channels by matching the richness of the communication channel (i.e., availability of immediate feedback, multiple cues, focus on personal interests, and variety of language) with the equivocality of task (Daft and Lengel 1983). When the content of communication is ambiguous, people are likely to choose rich a communication channel, such as person-to-person communication. When the content of communication is clear, people will choose lean media, such as mediated communication. The similarities of the two mediated communication networks in terms of their alter composition and high turnover rates could be due to decreased ambiguity in communication content and reduced time constraints in the mediated communication networks. People may turn to different people for clarity and establish temporary relationships. This could explain why the person-to-person network has a relatively higher turnover and its alter composition has less overlap with the mediated communication networks. It is also important to keep in mind that the results on in-person interactions rely on Bluetooth measurements, which likely constitute a mix of purposeful interactions, chance encounters with previously known members of an individual's social network, structured interactions such as being in the same classroom, or simple chance encounters such as passing by one another in a hallway.

Despite the differences in social interaction patterns between the person-to-person communication network and the mediated communication networks, similarities across different communication modes exist as well. We found that social signatures of both person-to-person and mediated communication networks are persistent over time and consistent across channels, even though the rate of turnover of network members is high, which is consistent with previous research (Saramäki et al. 2014; Aledavood et al. 2015; Heydari et al. 2018). The high level of turnover indicates that the persistence of social signatures is not attributable to relatively static network membership. The consistency of social signatures across different communication modes indicates that the consistency is not due to the channel of communication. The similarity between a given individual's social signatures of different social networks rather than the similarity between the social signatures of different people in the same social network further indicates that the potential reasons of persistence and consistency of social signatures 
might be within individuals. Psychologists have proposed several explanations that may explain the persistence of social signatures over time. The first is the cognitive constraint hypothesis, which argues that people have limited cognitive resources to spend on social interactions (Bernard and Killworth 1973; Saramäki et al. 2014). Each individual's cognitive constraints are consistent over time, but vary across individuals. Similarly, time is also a limited resource with respect to social interactions (Roberts and Dunbar 2011). The individual cognitive and time constraints are reflected as distinct social signature patterns. Another explanation is the social brain hypothesis that assumes the evolution of large brains is driven by the demand of coping with complex social lives (Dunbar 1998; Arnaboldi et al. 2015). Different volumes of core brain regions may determine the number of individuals people can maintain at each level of emotional closeness (Saramäki et al. 2014). Future studies may examine whether these mechanisms could explain the persistence and consistency of human social signatures using experiments rather than observational studies.

The evidence of the persistence and consistency of social signatures advances literature in the following ways. First, our study provides a broader understanding of social signatures by showing that the persistence of social signatures exists not only in mediated networks, as showed in previous studies (Saramäki et al. 2014; Heydari et al. 2018), but also in in-person networks. We also show that the social signatures within one individual are consistent across the in-person network and the mediated networks, but different from other individuals' social signatures. Second, by using so far the largest and most complex social network data to examine questions relating to social signatures, our study meaningfully advances the generalizability of studies in this area. Third, our study shows that researchers do not need a long time period to observe the persistence and consistency of social signatures. Within a month, we observed evidence of the persistence and consistency of social signatures, which is a substantially shorter period of observation than in other studies. This suggests that researchers may be able to incorporate social signatures to research on phenomena that take place in a short period of time. Further, given the substantial resources required to follow a full social network over an extended period of time, being able to examine processes related to social signatures in shorter periods of time will make research in this area more accessible. Fourth, the persistence and consistency of social signatures indicate invariant social network structures, which can be used to cast basic theoretical assumptions in formal models of social networks.

The persistence and consistency of social signatures give scholars valuable opportunities to make inferences for other networks when the information on a given network is known. Scholars have successfully used the frequency of online social interactions to predict the offline tie strength (Jones et al. 2013; Sapiezynski et al. 2018) and have used the strength of Bluetooth signals to predict online relationships (Sekara and Lehmann 2014). Our study suggests that similarities between the highly overlapping mediated networks may enable researchers to make inferences from one mediated network about another. Our findings also suggest that using the frequency of online social interactions to predict offline tie strength need to consider the alter composition of each social network. The alter composition of the person-to-person network is quite different from that of the mediated network due to high turnover. An individual who is a strong tie in the person-to-person network may not be a strong tie with the same ego through phone 
calls or text messages. Therefore, even though our study suggested that, for a given individual, their social signatures patterns in the person-to-person network and the mediated networks are similar to each other, scholars should consider differences in alter composition before making inferences about tie strength from one network to another.

The persistence and consistency of social signatures may also contribute to research about attitudes and behaviors. External environments surrounding individuals, such as their social networks, can shape, activate, or restrict the attitudes or behaviors of individuals (Hafner-Burton and Montgomery 2010). Our study further supports prior research showing that social signatures are persistent over time, which may improve a major limitation of cross-sectional studies on how egocentric networks may influence individuals' attitudes and behaviors (Eveland and Hively 2009; Hively and Eveland 2009; Eveland et al. 2013; Eveland and Hutchens 2013; Song and Eveland 2015; Eveland and Shen 2021), namely that the findings may illuminate short-term relationships but whether those same relationships hold in the long term is unknown. Our findings suggest that individuals' egocentric allocations of social resources, specifically social signatures, are persistent across time, indicating that the findings from cross-sectional studies may have long-term implications.

Our findings about the integrated multi-layer network also shed light on studies about the social contagion and social influence processes. To achieve successful information transmission or social influence, simple contagion requires multiple exposures to the same source, whereas complex contagion requires multiple sources of exposure (Centola 2018). Thus, high turnover rates may inhibit simple contagion but could encourage complex contagion. The relatively low turnover rates may inhibit complex contagion processes as people have relatively fewer chances to be exposed to different sources of the same content. We found that person-to-person networks have relatively higher turnover rates compared to mediated networks, which indicates mediated networks may be more likely to foster simple contagion processes and person-to-person networks may be more conducive to the complex contagion processes.

Our study also has important limitations. First, though our study has the largest sample size using multiple communication channels to study social signatures to date, we are uncertain of the extent to which our study can be generalized to other populations and over time. Like the original study on social signatures (Saramäki et al. 2014), we also used convenience student sample. The student sample limits the generalizability of our study. Though we believe it is important to know how young adults form social connections via different communication channels, we are unsure whether these findings would be generalized to other populations. Future studies may want to examine whether social signatures hold the property of persistence and consistency among other populations.

Second, we used the Bluetooth data as a physical proximity indicator of person-toperson social interactions. The collection of sensor data (e.g., call records, text logs, or Bluetooth proximity logs) to study human social behaviors has been termed "reality mining" (Eagle and Pentland 2006). Using these digital records, researchers can accurately detect participants' social networks and regular patterns in daily activity (Eagle and Pentland 2006). Other examples of using mobile phone records for social sensing include the friends and family study in a residential community (Aharony et al. 2011) and the GroupUs study to predict human interaction types (Do and Gatica-Perez 2011). 
Though the use of Bluetooth data to study social interactions has gained traction, we acknowledge the lack of precision of using Bluetooth data as a proxy of in-person social interactions. On the one hand, Bluetooth data is relatively noisy so that occasionally a Bluetooth device may not detect all nearby devices in a scan (Do and Gatica-Perez 2011). More importantly, the nature of Bluetooth data cannot tell individual choices from social circumstances. For example, we cannot know whether two students are physically close to each other because they intentionally choose to interact or they happen to attend the same class, among other reasons they may be physically proximate. Individual choices may reflect the underlying social signatures, whereas social circumstances (e.g., attending the same class) may bring in confounds to the results. Therefore, the person-to-person interaction results should be interpreted with caution given the uncertainty of the measurement strategy.

In summary, our work found that social signatures are persistent over time and consistent across different communication channels for each individual. The social signatures in different time periods and communication channels within the same individual are similar to each other rather than similar to a random individual's social signature in the same communication channel. The two mediated networks (i.e., phone call network and text message network) are highly overlapped, whereas the person-to-person network has relatively low overlap with the two mediated networks. The two mediated networks also display relatively low turnover rates compared to the person-to-person network. We expect that our work will advance human understanding of the dynamic social interaction patterns across different communication modes. We also expect our work can have implications for empirical studies on attitudes and behaviors, diffusion of information, among other important network phenomena.

\section{Abbreviations}

CNS: Copenhagen Networks Study; J: Jaccard Index; JSD: Jensen-Shannon distance.

Acknowledgements

Not applicable.

Authors' contributions

All authors designed the research, discussed results, and contributed to preparation of the manuscript. Both authors read and approved the final manuscript.

Funding

The authors do not declare any funding.

Availability of data and materials

Data and code required to reproduce this paper's results will be made available online through Harvard Dataverse.

\section{Declarations}

Competing interests

The authors declare no competing interests.

Received: 24 August 2021 Accepted: 5 February 2022

Published online: 24 February 2022

References

Aharony N, Pan W, Ip C, Khayal I, Pentland A (2011) Social FMRI: investigating and shaping social mechanisms in the real world. Pervasive Mob Comput 7(6):643-659

Ahn Y-Y, Bagrow JP, Lehmann S (2010) Link communities reveal multiscale complexity in networks. Nature 466(7307):761-764 
Aledavood T, López E, Roberts SG, Reed-Tsochas F, Moro E, Dunbar Rl, Saramäki J (2015) Daily rhythms in mobile telephone communication. PLOS ONE 10(9):e0138098

Aledavood T, López E, Roberts SG, Reed-Tsochas F, Moro E, Dunbar Rl, Saramäki J (2016) Channel-specific daily patterns in mobile phone communication. In: Proceedings of ECCS 2014. Springer, pp 209-218

Alessandretti L, Sapiezynski P, Sekara V, Lehmann S, Baronchelli A (2018) Evidence for a conserved quantity in human mobility. Nat Hum Behav 2(7):485-491

Arnaboldi V, Guazzini A, Passarella A (2013) Egocentric online social networks: analysis of key features and prediction of tie strength in facebook. Comput Commun 36(10-11):1130-1144

Arnaboldi V, Passarella A, Conti M, Dunbar RI (2015) Online social networks: human cognitive constraints in Facebook and Twitter personal graphs. Elsevier, Amsterdam

Bernard HR, Killworth PD (1973) On the social structure of an ocean-going research vessel and other important things. Soc Sci Res 2(2):145-184

Borgatti SP, Mehra A, Brass DJ, Labianca G (2009) Network analysis in the social sciences. Science 323(5916):892-895

Cattuto C, Van den Broeck W, Barrat A, Colizza V, Pinton J-F, Vespignani A (2010) Dynamics of person-to-person interactions from distributed RFID sensor networks. PLoS ONE 5(7):e11596

Centola D (2018) How behavior spreads: the science of complex contagions, vol 3. Princeton University Press, Princeton

Daft RL, Lengel RH (1983) Information richness. A new approach to managerial behavior and organization design. Technical report, Texas A and M University College Station Coll of Business Administration

Do TMT, Gatica-Perez D (2011) Groupus: smartphone proximity data and human interaction type mining. In: 2011 15th annual international symposium on wearable computers. IEEE, pp 21-28

Dunbar RI (1998) The social brain hypothesis. Evolut Anthropol Issues News Rev 6(5):178-190

Dunbar RI (2014) The social brain: psychological underpinnings and implications for the structure of organizations. Curr Dir Psychol Sci 23(2):109-114

Dunbar Rl, Spoors M (1995) Social networks, support cliques, and kinship. Hum Nat 6(3):273-290

Dunbar Rl, Arnaboldi V, Conti M, Passarella A (2015) The structure of online social networks mirrors those in the offline world. Soc Netw 43:39-47

Eagle N, Pentland AS (2006) Reality mining: sensing complex social systems. Pers Ubiquit Comput 10(4):255-268

Eveland WP Jr, Hively MH (2009) Political discussion frequency, network size, and "heterogeneity" of discussion as predictors of political knowledge and participation. J Commun 59(2):205-224

Eveland WP Jr, Hutchens MJ (2013) The role of conversation in developing accurate political perceptions: a multilevel social network approach. Hum Commun Res 39(4):422-444

Eveland WP Jr, Shen F (2021) Cross-national variation in political network size, distribution, and prediction. Soc Netw 66:100-113

Eveland WP Jr, Hutchens MJ, Morey AC (2013) Political network size and its antecedents and consequences. Polit Commun 30(3):371-394

Fuchs B, Sornette D, Thurner S (2014) Fractal multi-level organisation of human groups in a virtual world. Sci Rep 4:6526

Garcia-Herranz M, Moro E, Cebrian M, Christakis NA, Fowler JH (2014) Using friends as sensors to detect global-scale contagious outbreaks. PLoS ONE 9(4):e92413

Grabowicz PA, Ramasco JJ, Moro E, Pujol JM, Eguiluz VM (2012) Social features of online networks: the strength of intermediary ties in online social media. PLoS ONE 7(1):e29358

Hafner-Burton EM, Montgomery AH (2010) Centrality in politics: how networks confer power

Heydari S, Roberts SG, Dunbar Rl, Saramäki J (2018) Multichannel social signatures and persistent features of ego networks. Appl Netw Sci 3(1):8

Hill RA, Dunbar RI (2003) Social network size in humans. Hum Nat 14(1):53-72

Hively MH, Eveland WP Jr (2009) Contextual antecedents and political consequences of adolescent political discussion, discussion elaboration, and network diversity. Polit Commun 26(1):30-47

Holme P, Saramäki J (2012) Temporal networks. Phys Rep 519(3):97-125

Holt-Lunstad J, Smith TB, Layton JB (2010) Social relationships and mortality risk: a meta-analytic review. PLoS Med 7(7):e1000316

House JS, Landis KR, Umberson D (1988) Social relationships and health. Science 241(4865):540-545

Jones JJ, Settle JE, Bond RM, Fariss CJ, Marlow C, Fowler JH (2013) Inferring tie strength from online directed behavior. PLOS ONE 8(1):e52168

Kafsi M, Kazemi E, Maystre L, Yartseva L, Grossglauser M, Thiran P (2013) Mitigating epidemics through mobile micromeasures. arXiv preprint arXiv:1307.2084

Koltsova OY, Mararitsa LV, Terpilovskii MA, Sinyavskaya YE (2021) Social signature in an online environment: stability and cognitive limits. Comput Hum Behav 122:106856

Laurent G, Saramäki J, Karsai M (2015) From calls to communities: a model for time-varying social networks. Eur Phys J B 88(11):301

Lewis K, Gonzalez M, Kaufman J (2012) Social selection and peer influence in an online social network. Proc Natl Acad Sci 109(1):68-72

Lin Y-R, Chi Y, Zhu S, Sundaram H, Tseng BL (2009) Analyzing communities and their evolutions in dynamic social networks. ACM Trans Knowl Discov Data 3(2):1-31

Liu J-G, Guo Q, Zhang Y-C et al (2018) Social signature identification of dynamical social networks. Physica A 508:213-222

Mac Carron P, Kaski K, Dunbar R (2016) Calling Dunbar's numbers. Soc Netw 47:151-155

Manninen S, Tuominen L, Dunbar RI, Karjalainen T, Hirvonen J, Arponen E, Hari R, Jääskeläinen IP, Sams M, Nummenmaa L (2017) Social laughter triggers endogenous opioid release in humans. J Neurosci 37(25):6125-6131

McPherson M, Smith-Lovin L, Cook JM (2001) Birds of a feather: homophily in social networks. Ann Rev Sociol 27(1):415-444

Milardo RM, Johnson MP, Huston TL (1983) Developing close relationships: changing patterns of interaction between pair members and social networks. J Pers Soc Psychol 44(5):964 
Miritello G, Moro E, Lara R, Martínez-López R, Belchamber J, Roberts SG, Dunbar RI (2013) Time as a limited resource: communication strategy in mobile phone networks. Soc Netw 35(1):89-95

Mones E, Stopczynski A, Lehmann S (2017) Contact activity and dynamics of the social core. EPJ Data Sci 6(1):1-16

Nanavati AA, Singh R, Chakraborty D, Dasgupta K, Mukherjea S, Das G, Gurumurthy S, Joshi A (2008) Analyzing the structure and evolution of massive telecom graphs. IEEE Trans Knowl Data Eng 20(5):703-718

Onnela J-P, Saramäki J, Hyvönen J, Szabó G, Lazer D, Kaski K, Kertész J, Barabási A-L (2007) Structure and tie strengths in mobile communication networks. Proc Natl Acad Sci 104(18):7332-7336

Palla G, Derényi I, Farkas I, Vicsek T (2005) Uncovering the overlapping community structure of complex networks in nature and society. Nature 435(7043):814-818

Palla G, Barabasi A-L, Vicsek T (2007) Quantifying social group evolution. Nature 446:664-667

Powell J, Lewis PA, Roberts N, Garcia-Finana M, Dunbar RI (2012) Orbital prefrontal cortex volume predicts social network size: an imaging study of individual differences in humans. Proc R Soc B Biol Sci 279(1736):2157-2162

Roberts SG, Dunbar RI (2011) Communication in social networks: effects of kinship, network size, and emotional closeness. Pers Relat 18(3):439-452

Rosvall M, Esquivel AV, Lancichinetti A, West JD, Lambiotte R (2014) Memory in network flows and its effects on spreading dynamics and community detection. Nat Commun 5(1):1-13

Sapiezynski P, Stopczynski A, Wind DK, Leskovec J, Lehmann S (2018) Offline behaviors of online friends. arXiv preprint arXiv:1811.03153

Sapiezynski P, Stopczynski A, Lassen DD, Lehmann S (2019) Interaction data from the Copenhagen networks study. Sci Data 6(1):1-10

Saramäki J, Moro E (2015) From seconds to months: an overview of multi-scale dynamics of mobile telephone calls. Eur Phys J B 88(6):164

Saramäki J, Leicht EA, López E, Roberts SG, Reed-Tsochas F, Dunbar RI (2014) Persistence of social signatures in human communication. Proc Natl Acad Sci 111(3):942-947

Schulman I (2021) Data-driven study of social signatures in different channels. Master's thesis, Aalto University

Sekara V, Lehmann S (2014) The strength of friendship ties in proximity sensor data. PLoS ONE 9(7):e100915

Sekara V, Stopczynski A, Lehmann S (2016) Fundamental structures of dynamic social networks. Proc Natl Acad Sci 113(36):9977-9982

Song H, Eveland WP Jr (2015) The structure of communication networks matters: how network diversity, centrality, and context influence political ambivalence, participation, and knowledge. Polit Commun 32(1):83-108

Stiller J, Dunbar RI (2007) Perspective-taking and memory capacity predict social network size. Soc Netw 29(1):93-104

Stopczynski A, Sekara V, Sapiezynski P, Cuttone A, Madsen MM, Larsen JE, Lehmann S (2014) Measuring large-scale social networks with high resolution. PLoS ONE 9(4):e95978e95978

Szabó G, Barabasi A-L (2006) Network effects in service usage. arXiv preprint arXiv:physics/0611177

Wang Y, Faloutsos M, Zang H (2013) On the usage patterns of multimodal communication: countries and evolution. In: 2013 proceedings IEEE INFOCOM. IEEE, pp 3135-3140

Zhang J, Litvinova M, Liang Y, Wang Y, Wang W, Zhao S, Wu Q, Merler S, Viboud C, Vespignani A et al (2020) Changes in contact patterns shape the dynamics of the covid-19 outbreak in china. Science

\section{Publisher's note}

Springer Nature remains neutral with regard to jurisdictional claims in published maps and institutional affiliations.

\section{Submit your manuscript to a SpringerOpen ${ }^{\oplus}$ journal and benefit from:}

- Convenient online submission

Rigorous peer review

- Open access: articles freely available online

- High visibility within the field

- Retaining the copyright to your article

Submit your next manuscript at $\boldsymbol{\nabla}$ springeropen.com 